\title{
MAGNETIC PHASE TRANSITION OF AMORPHOUS ALLOYS FeNiSiB
}

\author{
K. BrzózKa, M. Gawroński and K. Jezuita \\ Department of Physics, Technical University, Malczewskiego 22a, 26-600 Radom, Poland
}

\begin{abstract}
Static and dynamical behaviour of amorphous ferromagnets FeNiSiB was investigated by the Mössbauer spectroscopy and magnetostatic methods below and above $T_{\mathrm{C}}$. The distributions of hyperfine magnetic field in the ferromagnetic phase and quadrupole splitting in the paramagnetic one were extracted by means of the constrained Hesse-Rübartch method. The static critical exponents were determined using the power laws and the Kouvel-Fisher method. The results of the Mössbauer investigations were compared with those obtained by magnetic measurements.
\end{abstract}

PACS numbers: $64.60 .-\mathrm{i}, 75.50 .-\mathrm{y}, 76.80 .+\mathrm{y}$

\section{Introduction}

With the aim of studies of the magnetic phase transition in amorphous FeNiSiB ferromagnets two complementary experimental technics were used: the Mössbauer spectroscopy and bulk magnetization measurements by use of a magnetic balance.

The first, microscopic method is sensitive to local fluctuations of the hyperfine parameters: hyperfine magnetic field, quadrupole splitting and isomer shift. Static critical exponent $\beta$ is related to the average hyperfine magnetic field. The dynamical effects can be also observed.

The second method is macroscopic in this sense that it allows to measure average magnetic moment. It needs presence of external magnetic field, then the static critical exponents $\beta, \gamma, \delta$ have to be determined by analyzing of magnetic equation of state.

\section{Experimental}

Amorphous ferromagnetic alloys FeNiSiB in form of ribbons with thickness about $30 \mu \mathrm{m}$, prepared by the conventional melt spinning method were investigated. Most of them were produced at the Institute of Materials Science and Engineering (Technical University of Warsaw).

The series of the Mössbauer measurements were performed in the temperature range $300-750 \mathrm{~K}$. The conventional transmission geometry and the absorber 
technic were used and the temperature stability over $24 \mathrm{~h}$ (controlled by computer) was better than $0.15 \mathrm{~K}$.

The magnetization $M$ was measured as a function of temperature and external magnetic field, in the range $500 \mathrm{Oe} \leq H_{\text {ext }} \leq 11 \mathrm{kOe}$.

\section{Critical behaviour from the Mössbauer studies}

A review of the more common, model independent methods to obtain hyperfine parameter distributions $P(x)$ in the Mössbauer effect spectroscopy has recently been published $[1,2]$. During studies of amorphous FeNiSiB ferromagnets $[3-6]$ we have used as well the matrix method $[7,8]$ as the expansion of $P(x)$ over a function set [9-11]. But not all the methods are convenient in the critical region. That is just why we have recently followed the Hesse-Rübartch method $[7,8]$. In our case the hyperfine parameter $x$ is the local hyperfine magnetic field or the quadrupole splitting. We present here only some important results related to the magnetic phase transition.

In these amorphous alloys the continuous, well-defined critical point occurs. Assuming that below the Curie temperature $T_{C}$ the local magnetic hyperfine field is proportional to the local magnetization, the temperature dependence of the effective exponent $\beta_{\text {eff }}$ and the ratio of the width of hyperfine field distribution to the average hyperfine field $\Delta H / H_{\text {mean }}$ were analyzed. The variation of the effective exponent $\beta_{\text {eff }}$ shown in Fig. 1 is in agreement with other experimental data and theory [12]. The quantity $\Delta H / H_{\text {mean }}$ slightly increases with increasing

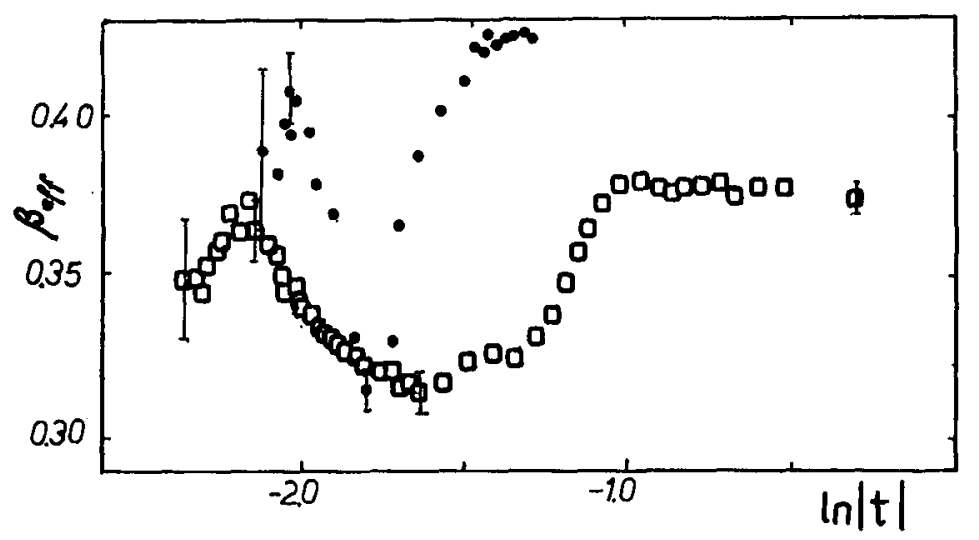

Fig. 1. Effective exponent $\beta_{\text {eff }}$ obtained from the Mössbauer spectroscopy: $\square \mathrm{Fe}_{35} \mathrm{Ni}_{40} \mathrm{Si}_{10} \mathrm{~B}_{15}, \circ \mathrm{Fe}_{45} \mathrm{Ni}_{30} \mathrm{Si}_{10} \mathrm{~B}_{15}$.

temperature and reaches a maximum just below $T_{\mathrm{C}}[4,5]$ as it was predicted by Fähnle and Herzer [13], by means of the self-consistent correlated molecular field theory developed for disordered systems.

The Mössbauer line broadening just above $T_{\mathrm{C}}$ was observed, which could be interpreted as a critical effect [13]. 


\section{Critical behaviour from magnetic measurements}

To derive the zero-field properties like spontaneous magnetization $M_{\mathbf{s}}$ and initial susceptibility $\chi_{0}$ the modified asymptotic analysis method AAII [12] was used. This method is based on the magnetic equation of state:

$$
(H / M)^{1 / \gamma}=a t+b M^{1 / \beta}
$$

which is postulated to be valid in the asymptotic critical region. Here $t=$ $\left(T-T_{\mathrm{C}}\right) / T_{\mathrm{C}}$ is the reduced temperature and $H$ is the internal magnetic field ( $H=H_{\text {ext }}-H_{\mathrm{d}}, H_{\mathrm{d}}-$ demagnetization field). We make use of the iteration procedure, starting with $\beta=0.4$ and $\gamma=1.4$ and computing values of $M_{\mathbf{s}}, \chi_{0}$ from the intercepts of the parallel straight-line isotherms in modified Arrot-Noakes plot $\left(M^{1 / \beta}\right.$ vs. $\left.(H / M)^{1 / \gamma}\right)$. The values of exponents $\beta$ and $\gamma$ were derived by the Kouvel-Fisher method based on the relations

$$
\begin{aligned}
& X(T)=\chi_{0}^{-1}\left(\mathrm{~d} \chi_{0}^{-1} / \mathrm{d} T\right)^{-1}=\left(T-T_{\mathrm{C}}\right) / \gamma, \\
& Y(T)=M_{\mathrm{s}}\left(\mathrm{d} M_{\mathrm{s}} / \mathrm{d} T\right)^{-1}=\left(T_{\mathrm{C}}-T\right) / \beta,
\end{aligned}
$$

with fitting parameters $\beta, \gamma, T_{\mathrm{C}}$, as illustrated by Fig. 2. The exponent $\delta$ was

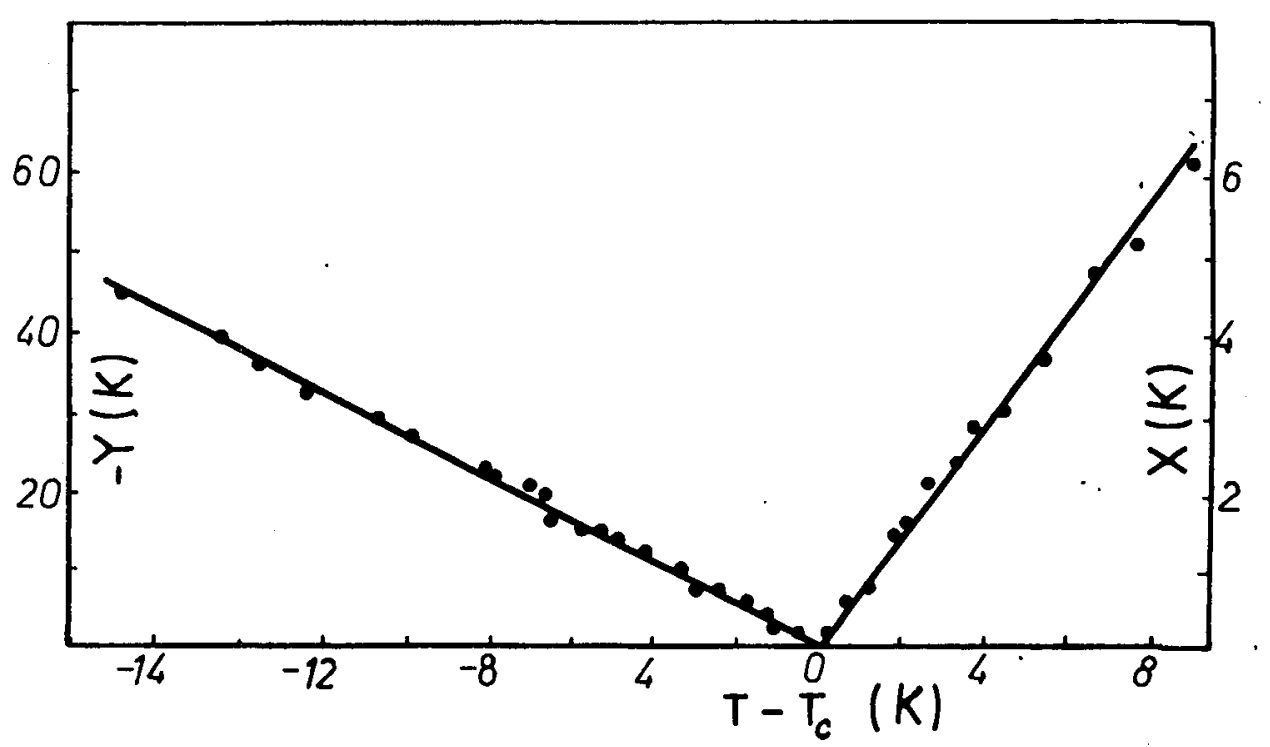

Fig. 2. Determination of $T_{\mathrm{C}}$ and critical exponents $\beta, \gamma$ by the Kouvel-Fisher method $\left(\mathrm{Fe}_{35} \mathrm{Ni}_{40} \mathrm{Si}_{10} \mathrm{~B}_{15}\right)$.

determined from the slope of the isotherms $M(H)$ in the critical region. The results of exponents $\beta, \gamma, \delta$ and $\delta_{0}, \alpha_{0}$ computing from the scaling relations are reported in Table $\mathbf{I}$. 
TABLE I

Critical exponents obtained from magnetic measurements.

\begin{tabular}{l|l|c|c|c|c}
\hline \hline & \multicolumn{1}{|c|}{$\beta$} & $\gamma$ & $\delta$ & $\delta_{0}$ & $\alpha_{0}$ \\
\hline $\mathrm{Fe}_{35} \mathrm{Ni}_{40} \mathrm{Si}_{10} \mathrm{~B}_{15}$ & $0.353(15)$ & $1.47(4)$ & $5.20(10)$ & $5.26(25)$ & $-0.18(7)$ \\
$\mathrm{Fe}_{40} \mathrm{Ni}_{35} \mathrm{Si}_{10} \mathrm{~B}_{15}$ & $0.354(15)$ & $1.45(4)$ & $5.16(10)$ & $5.23(25)$ & $-0.16(7)$ \\
$\mathrm{Fe}_{45} \mathrm{Ni}_{30} \mathrm{Si}_{10} \mathrm{~B}_{15}$ & $0.40(2)$ & $1.48(5)$ & $4.90(20)$ & $4.70(40)$ & $-0.28(9)$ \\
$\mathrm{Fe}_{40} \mathrm{Ni}_{40} \mathrm{Si}_{13} \mathrm{~B}_{7}$ & $0.358(15)$ & $1.50(4)$ & $5.30(10)$ & $5.19(25)$ & $-0.21(7)$
\end{tabular}

\section{Conclusions}

Compliance of results obtained by the Mössbauer spectroscopy and those derived from magnetic measurements (Table II) implies that a linear relationship between the average hyperfine magnetic field and the magnetization is fulfilled in the critical region.

\section{TABLE II}

The results obtained by the Mössbauer spectroscopy and from bulk magnetization measurements ( $A_{0}$ and $A$ are order parameter at room temperature and its critical amplitude).

\begin{tabular}{l|c|c|c|c|c|l}
\hline \hline & \multicolumn{3}{|c|}{$A$} & \multicolumn{2}{c|}{$A / A_{0}$} & \multicolumn{2}{c}{$\beta$} \\
\cline { 2 - 7 } & MS [kGs] & BM [emu/g] & MS & BM & MS & BM \\
\hline $\mathrm{Fe}_{40} \mathrm{Ni}_{40} \mathrm{Si}_{13} \mathrm{~B}_{7}$ & 275 & 102 & 1.40 & 1.41 & 0.370 & 0.358 \\
$\mathrm{Fe}_{35} \mathrm{Ni}_{40} \mathrm{Si}_{10} \mathrm{~B}_{15}$ & 285 & 100 & 1.43 & 1.35 & 0.355 & 0.353 \\
$\mathrm{Fe}_{40} \mathrm{Ni}_{40} \mathrm{Si}_{10} \mathrm{~B}_{15}$ & - & 117 & - & 1.25 & - & 0.354 \\
$\mathrm{Fe}_{45} \mathrm{Ni}_{30} \mathrm{Si}_{10} \mathrm{~B}_{15}$ & 360 & 130 & 1.20 & 1.24 & 0.395 & 0.40
\end{tabular}

The Mössbauer spectra analysis demonstrates that the sharpness of the magnetic phase transition in $\mathrm{FeNiSiB}$ amorphous ferromagnets is not affected by the quenched disorder. The values of the static critical exponents are close to the homogeneous three-dimensional model and satisfy the scaling relations, in conformity with the Harris criterion and the predictions of the renormalization group calculations $[12,14]$. This suggests the dominant role of the exchange interactions in the critical region.

The authors wish to thank Professor H. Szymczak and Dr. R. Puźniak for stimulating discussions and the help in realization of the magnetic measurements at the Institute of Physics of the Polish Academy of Sciences.

\section{References}

[1] G. Le Caër, R.A. Brand, Hyperfine Interact. 71, 1507 (1992).

[2] D.L. Nagy, U. Röhlich, Hyperfine Interact. 66, 105 (1991). 
[3] K. Brzózka, K. Jezuita, J. Szlanta, in: Magnetic Properties of Amorphous Metals, Ed. A. Hernando, V. Madurga, M.C. Sanches-Trujillo, M. Vazquez, Elsevier, Amsterdam 1987, p. 61.

[4] K. Brzózka, M. Gawroński, K. Jezuita, J. Szlanta, Acta Phys. Pol. A 72, 133 (1987).

[5] K. Brzózka, M. Gawroński, K. Jezuita, J. Szlanta, Acta Phys. Pol. A 76, 133 (1989).

[6] K. Brzózka, M. Gawroński, K. Jezuita, J. Szlanta, Hyperfine Interact. 59, 365 (1990).

[7] J. Hesse, A. Rübartch, J. Phys. E 7, 526 (1974).

[8] G. Le Caër, J. M. Dubois, J. Phys. E 12, 1083 (1979).

[9] B. Window, J. Phys. E 4, 401 (1971).

[10] I. Vincze, Solid State Commun. 5, 689 (1978).

[11] D.G. Raincourt, J.Y. Ping, Nucl. Instrum. Methods Phys. Res. B 58, 85 (1991).

[12] S.N. Kaul, J. Magn. Magn. Mater. 53, 5 (1985).

[13] P.M. Barret, Phys. Rev. B 34, 3513 (1986).

[14] C. Hohenemser, N. Rosov, A. Kleinhammes, Hyperfine Interact. 49, 267 (1989). 